\title{
Remotely controlled diffusion from magnetic liposome microgels
}

\author{
Jaroslav Hanuš, Martin Ullrich, Jiří Dohnal, Mandeep Singh, František Štěpánek ${ }^{*}$
}

Department of Chemical Engineering, Institute of Chemical Technology, Prague

Technicka 3, 16628 Prague 6, Czech Republic

${ }^{*}$ Corresponding author. E-mail: Frantisek.Stepanek@vscht.cz; Tel.: +420 220443236.

\begin{abstract}
The reversible, temperature-dependent change in the permeability of a phospholipid bi-layer has been used for controlling the diffusion rate of encapsulated molecular payload from liposomes. Liposomes were pre-loaded with a fluorescent dye and immobilized in calcium alginate hydrogel microparticles that also contained iron oxide nanoparticles. The composite microparticles were produced by a drop-on-demand ink-jet method. The ability of iron oxide nanoparticles to locally dissipate heat upon exposure to a radio-frequency (RF) alternating magnetic field was used to control the local temperature and therefore diffusion from the liposomes in a contactless way using RF coil. Several different release patterns were realised, including repeated on-demand release. The internal structure of the composite alginateliposome-magnetite microparticles was investigated and the influence of microparticle concentration on the heating rate determined. In order to achieve a temperature rise required for the liposome membrane melting, the concentration of alginate beads should be at least $25 \%$ of their maximum packing density for the nanoparticle concentration and specific absorption rate used.
\end{abstract}

Keywords: liposomes; alginate; iron oxide nanoparticle; ink-jet fabrication; radiofrequency heating.

\section{Introduction}

Liposomes (phospholipid vesicles) can encapsulate a broad range of molecules ${ }^{1}$ and they often serve as a protection from the environment potentially hostile to the encapsulated substances. Liposomes have traditionally served for achieving sustained, "passive", continuous release. Additionally, liposomes can be used for prolonging the circulation time of some drugs - in that case, PEGylated liposomes are used to avoid fast clearance or uptake by the reticuloendothelial system. ${ }^{2}$ In many cases, however, the possibility to release the liposome content on demand is desirable and strategies to control the release by various 
external stimuli are already reported. ${ }^{3-5}$ In this "active controlled release" scenario the liposomes should keep the encapsulated molecules inside for as long as possible without leakage, but once an external impulse (thermal, electrical, mechanical, change of $\mathrm{pH}$ etc.) is applied, a desired amount of the encapsulated chemical compound should be released. The scope of possible applications includes chemical microreactors ${ }^{6}$, smart diagnostic or drug carrying agents. ${ }^{7,8}$ However, not all methods for the externally controlled destabilization of liposomes are applicable in vivo or in situations where direct access to the liposomes is limited. One of the promising concepts that is applicable in such situations, uses energy provided by magnetic nanoparticles (MNPs) when exposed to an alternating magnetic field. ${ }^{9}$

Magnetic nanoparticles ${ }^{10}$ are already used in imaging (MRI) ${ }^{11}$, are tested in clinical studies for therapy (magnetic hyperthermia ${ }^{12}$ ) and can be also used in triggerable systems mentioned above. Although a variety of different materials and sizes can be used in principle, most studies are nowadays carried out with iron oxide nanoparticles (maghemite $\gamma-\mathrm{Fe}_{2} \mathrm{O}_{3}$ or magnetite $\mathrm{Fe}_{3} \mathrm{O}_{4}$ ) that are within the 5-30 nm size range. Such nanoparticles are reasonably biocompatible, can be stabilized in water suspension by using hydrophilic coating and have sufficient magnetic properties to respond to external magnetic field.

Efforts to combine magnetic nanoparticles and liposomes into the so-called magnetoliposomes have been recently reported. ${ }^{13-15}$ Nanoparticles with a hydrophobic coating have been integrated directly into the phospholipid bi-layer ${ }^{4}$ whereas hydrophilic nanoparticles have been encapsulated into the internal volume of liposomes. ${ }^{16,17}$ It is also possible to decorate the external liposome surface (or the place of contact of two liposomal membranes) by nanoparticles. ${ }^{3}$ Each approach has some advantages and limitations. The presence of MNPs directly in the lipid bi-layer makes it possible to destabilize the liposomes by physical movement of the nanoparticles rather than heat dissipation ${ }^{4}$ and lower nanoparticle concentrations should therefore be sufficient. However, the nanoparticle size needs to be precisely controlled and a special hydrophobic coating applied. ${ }^{18}$ On the other hand, hydrophilic MNPs are more readily available but their concentration within the liposome has to be relatively high in order to achieve the required temperature rise needed for the phase transition of the lipid bilayer.

A different concept is proposed in the present work whereby MNPs are not incorporated directly into the liposomes, but both are embedded within a larger carrier microparticle where liposomes serve as internal reservoirs with tunable size and properties, and MNPs have the role of stimulus responsive agents (Fig. 1). The main potential advantage of this approach is the ability to independently adjust the liposome and the MNP identity and concentration 
within the carriers and to use hydrophilic MNPs with a broader size distribution that are generally easier to synthesize. Alginate hydrogel - a biocompatible, easy to prepare material, whose interaction with liposome has already been studied ${ }^{19-21}$ can be used for the body of the mother particle. It was found that alginate reduces the stability of liposomes against leakage in case of phosphatidylcholine liposomes at room temperature and that by adding cholesterol this stability can be improved. In our recent work ${ }^{22}$ we have systematically investigated the cholesterol influence on the stability of liposomes against leakage during alginate gel formation. It was found that for optimum parameter settings (temperature, cholesterol content) the leakage from liposomes during ionic gelation of alginate does not exceed $10 \%$. It has also been shown that composite liposome-alginate microparticles can be produced by the drop-on-demand ink-jet technique ${ }^{23}$ and that liposomes are not destroyed during this process. In the present work, we will demonstrate the ability of such composite microparticles to respond to radiofrequency (RF) magnetic field by an increase of temperature and therefore controlled diffusion of encapsulated chemical payload from the liposomes. We will determine the relationship between the concentration of alginate beads and the achieved RF induced release rate. We also investigate the complex internal microstructure and liposome distribution within the gel microparticles.

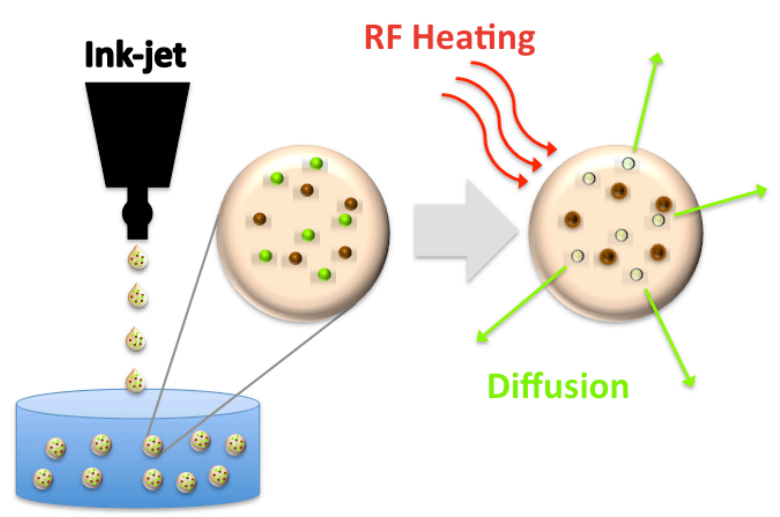

Fig. 1: Scheme of the liposome alginate magnetic bead fabrication and radiofrequency induced release of encapsulated chemical payload.

\section{Experimental}

\subsection{Chemicals}

Liposomes were prepared from 1,2-dipalmitoyl-sn-glycero-3-phosphocholine (DPPC) purchased from Corden Pharma LLC, Switzerland and from cholesterol (Chol) purchased from Sigma. Sephadex ${ }^{\circledR}$ G-50, surfactant Triton ${ }^{\circledR}$ X-100, Tris (2-Amino-2-hydroxymethylpropane-1,3-diol), CF [5(6)-carboxyfluorescein] were also provided by Sigma. Sodium 
alginate (mean chain length of 40,000 units) was purchased from SAFC®, chloroform, hydrochloric acid and calcium chloride were purchased from Lach-Ner, Czech Republic. Deionized water (specific conductivity $1.1 \mu \mathrm{S} . \mathrm{cm}^{-1}$ ) was filtered by Aqual 25 (Aqual, Czech Republic).

\subsection{Magnetic nanoparticles}

Hydrophilic magnetic nanoparticles stabilized by citrate ligands were prepared by the well known co-precipitation method ${ }^{24}$. Briefly, $1.0 \mathrm{~g}$ of $\mathrm{FeCl}_{3} \cdot 6 \mathrm{H}_{2} \mathrm{O}$ and $0.5 \mathrm{~g}$ of $\mathrm{FeCl}_{2} \cdot 4 \mathrm{H}_{2} \mathrm{O}$ were dissolved in $35 \mathrm{~mL}$ of deionised water and stirred for 5 minutes at $80{ }^{\circ} \mathrm{C}$ in a water bath. After 10 minutes, $5 \mathrm{~mL}$ of concentrated $\mathrm{NH}_{4} \mathrm{OH}$ was added at once into the solution. After 30 minutes of stirring, $1.00 \mathrm{~g}$ of tri-sodium citrate dissolved in $5 \mathrm{~mL}$ water was added to the above solution and the temperature was increased to $95{ }^{\circ} \mathrm{C}$ under vigorous stirring. After 90 minutes of stirring, a precipitate was collected by magnetic decantation, washed with water three times and dried in air at $80^{\circ} \mathrm{C}$, thus obtaining the citrate coated iron oxide nanoparticles. TEM (JEOL-1200EX microscope) and XRD (PANalytical X'pert PRO) were used to characterise the obtained nanoparticles (see Supporting Information). The mean size of the obtained from TEM measurements was $10 \mathrm{~nm}$ (number weighted). These nanoparticles were then resuspended in deionised water and filtration (450 nm Millipore filters) was used in order to remove larger aggregates. After filtration the volume-weighted hydrodynamic mean size of the nanoparticles in suspension was measured by dynamic light scattering (ZetaSizer Nano-ZS, Malvern Instruments, UK) and was found to be $15 \mathrm{~nm}$ (see Supporting Information).

\subsection{Radiofrequency heating}

The heating capabilities of MNPs were characterized by monitoring the time-dependent temperature rise of a $0.6 \mathrm{ml}$ MNPs suspension with defined concentration, exposed to alternating magnetic field (AMF). The $400 \mathrm{kHz}$ PowerCube generator (series 32/400, CEIA Power Systems, Italy) with a field amplitude of $20 \mathrm{mT}$ was employed as a source of radiofrequency magnetic field. Active isolating layer (in-house fabricated intermediate coil with independently conditioned medium) was used to shield the sample from direct heating by the magnetic coil, while a thermally insulated non-magnetic container was inserted inside. Temperature of the sample was measured by a fiber optic temperature sensor from Neoptix.

\subsection{Liposome preparation}


Liposomes were prepared by the Bangham method. $20 \mathrm{mg}$ of lipids (DPPC:Chol in 2:1 molar ratio) was dissolved in $2 \mathrm{ml}$ of chloroform and a rotary evaporator (IKA ${ }^{\circledR} \mathrm{RV} 10$ ) was used to gently evaporate the solvent at $65{ }^{\circ} \mathrm{C}$, while the pressure was progressively decreased to 200 mbar. Thin lipid film formed at the bottom of the flask was dried overnight under vacuum. In order to obtain liposomes, $3 \mathrm{~mL}$ of hydration medium heated to $65{ }^{\circ} \mathrm{C}$ was then added and the mixture was vortexed for 30 minutes. $50 \mathrm{mM}$ solution of $\mathrm{CF}$ in Tris-HCl buffer (170 mM, isotonic, pH 7.4) was used as the hydration medium. Extrusion method was employed to reduce the size and lamellarity of liposomes; 15 passes of extrusion (Avanti Mini-Extruder; Avanti Polar Lipids, USA) through a polycarbonate membrane were sufficient to obtain a narrow size distribution of liposomes; their mean size (measured by dynamic light scattering) corresponded to the pore size of the membrane (400 nm followed by another extrusion with $100 \mathrm{~nm}$ pores). The final liposome size measured by dynamic light scattering was around $100 \mathrm{~nm}$ for all experiments. Gel permeation chromatography (Sephadex ${ }^{\circledR}$ G-50 as a stationary phase in Tris-HCL buffer $10 \mathrm{mM}$ ) was then used to remove the non-entrapped carboxyfluorescein. The final liposome concentration (measured from the sample fluorescence after destroying the liposomes using Triton surfactant) was estimated to be approximately $1 \% \mathrm{v} / \mathrm{v}$.

\subsection{Alginate microgel formation and characterization}

Suspension of MNPs in deionized water ( $7 \mathrm{~g} / \mathrm{l})$ was first prepared and sodium alginate was then dissolved in this suspension so as to obtain a $2 \% \mathrm{w} / \mathrm{w}$ alginate concentration. This alginate - MNPs suspension was then mixed at a ratio of $1: 1(\mathrm{v} / \mathrm{v})$ with a suspension of liposomes purified by gel chromatography and stirred for 15 minutes. The obtained mixture was ink-jetted (ejection frequency $200-500 \mathrm{~Hz}$ ) into a $2 \%(\mathrm{w} / \mathrm{w})$ calcium chloride solution cooled by an ice bath, where the alginate chains were cross-linked by divalent calcium ions and formed gel microbeads. A piezoelectric drop-on-demand print-head type M5-ABP-01-806MX supplied by Microfab, Inc. (Plano, Texas, USA) has been used, coupled with a control unit type JetDrive III and a pressure controller type CT- PT-01 also supplied by Microfab, Inc. Then the microgel beads were separated from the $\mathrm{CaCl}_{2}$ solution by magnetic decantation, washed by deionized water and stored for further use. From the concentrations of liposomes $(0.5 \% \mathrm{v} / \mathrm{v})$ and MNPs $(3.5 \% \mathrm{w} / \mathrm{w})$ in the mixed suspension it can be estimated that one typical $50 \mu \mathrm{m}$ alginate bead would contain approximately 64,000 liposomes (100 nm mean diameter) and 87,000,000 magnetic nanoparticles (10 $\mathrm{nm}$ mean diameter). The internal structure of the ink-jet printed microgel beads was visualized by Laser Scanning Confocal Microscopy (LSCM) and wide-field optical and fluorescent microscopy. The Olympus 
Fluoview FV-1000 confocal system was used. The excitation wavelength of $488 \mathrm{~nm}$ was used for the carboxyfluorescein dye.

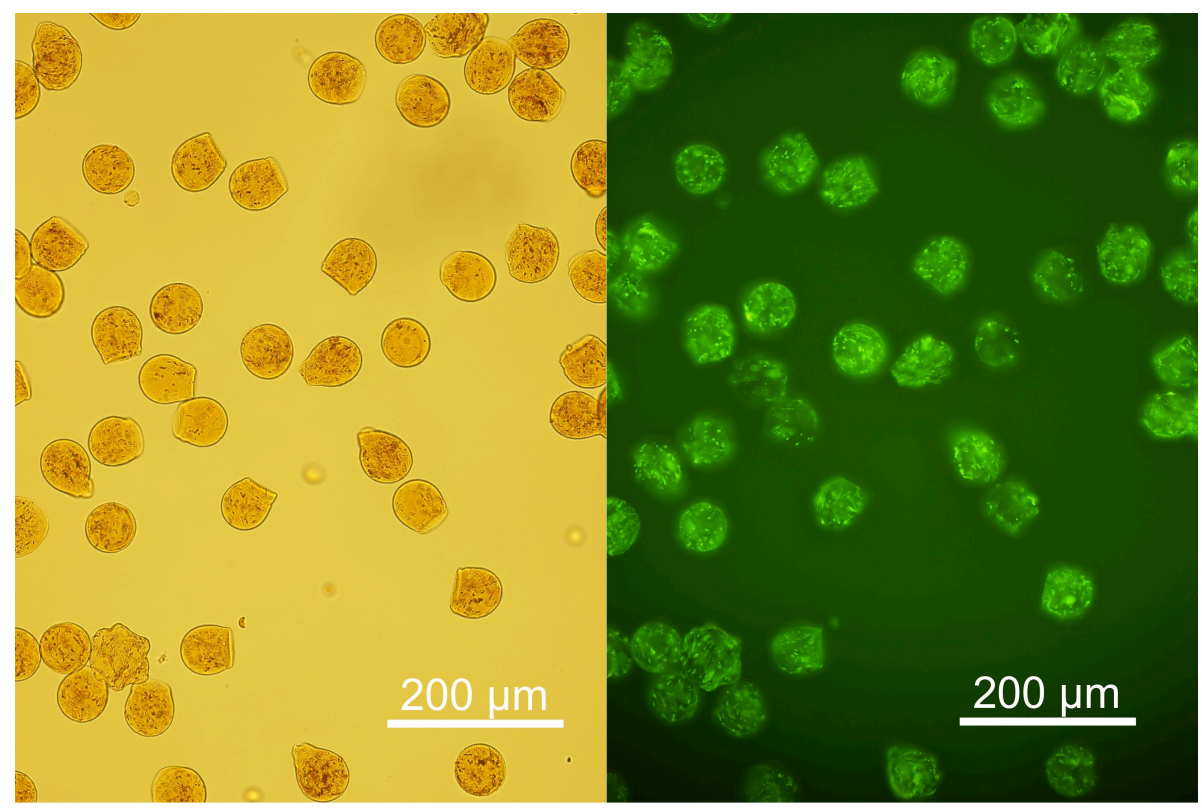

Fig. 2: Optical (left) and wide field fluorescent (right) image of the liposome-alginatemagnetic beads.

\subsection{Release kinetics measurement}

Carboxyfluorescein (CF) was chosen as a model dye for evaluating the release kinetics profiles. Fluorescence was measured by Cary Eclipse Fluorescence Spectrometer, Agilent Technologies, USA. 5-20 $\mu$ l of microgel suspension was dispersed in $3 \mathrm{ml}$ of isotonic $10 \mathrm{mM}$ Tris-HCl buffer. Surfactant Triton ${ }^{\circledR}$ X-100 (40 $\mu$ l of $10 \%$ solution) was used at the end of measurement to destroy the liposomes and determine the total amount of entrapped dye. The released amount was normalized with respect to this value. The efficacy of Triton to penetrate inside the beads and destroy all the liposomes was confirmed by heating experiments, where the beads (with or without Triton) were heated to $70{ }^{\circ} \mathrm{C}$ for 60 minutes and the sample fluorescence was measured after cooling back down to $25{ }^{\circ} \mathrm{C}$. There was no systematic increase of fluorescence values when compared to those obtained by using only Triton, which implies that the liposome destruction by Triton was essentially complete. For temperaturedependent measurements, the fluorescence intensity was recalculated to $25{ }^{\circ} \mathrm{C}$ using a previously obtained calibration curve. Temperature-dependent release kinetics experiments were done in two different ways - either by direct temperature control of the sample in a thermostated cuvette holder within the fluorescent spectrophotometer, or by the application of 
alternating magnetic field to a sample outside of the spectrophotometer and sampling for offline analysis.

\section{Results and discussion}

\subsection{Structure of Liposome-Alginate-Magnetic Beads (LAMBs)}

The microgel beads obtained from the ink-jet technique had a size range between $40-80 \mu \mathrm{m}$ (Fig. 2). Most of the beads have a spherical or drop-like shape and they stay well separated from each other in an aqueous medium - generally they resemble liposome-free alginate microparticles reported $\mathrm{in}^{25}$. However, due to their MNPs content they are strongly attracted by an external magnetic field (Fig. 3), which can be advantageous for their guiding to a target and for their local accumulation and concentration.

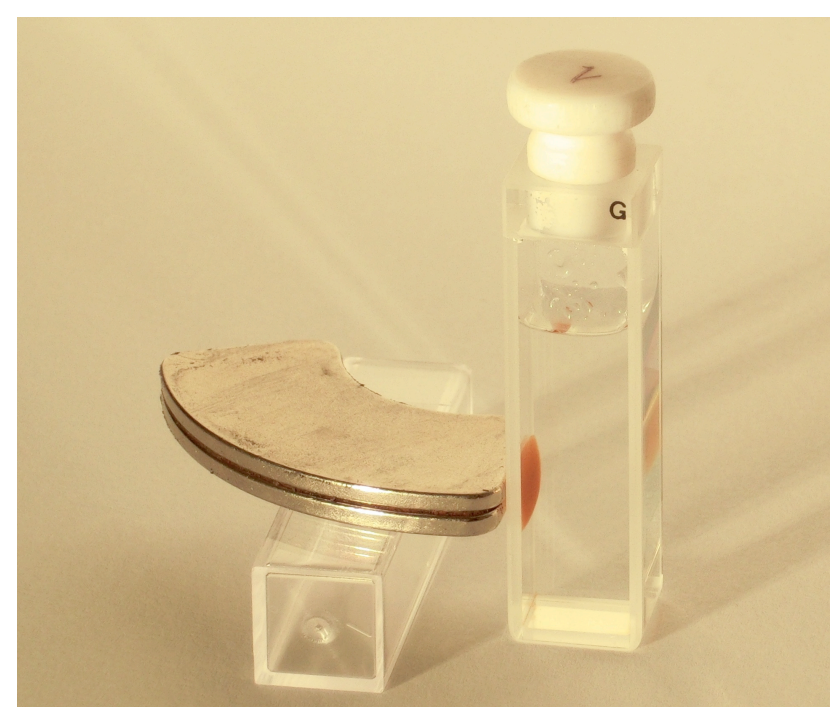

Fig. 3: Demonstration of magnetic properties of the LAMBs.

Confocal microscopy has been used to inspect the inner structure of LAMBs. When we compared typical confocal images of LAMBs with those obtained for macroscopic alginate beads also containing carboxyfluorescein in embedded liposomes ${ }^{22}$, there was a striking difference in liposome distribution. While in macroscopic beads the liposomes are distributed homogenously and individually throughout the volume of the bead, in ink-jet fabricated microbeads there are visible liposome streams (Fig 4a). Our first hypothesis was that this effect might be caused by the interaction of liposomes with the magnetic nanoparticles and then with alginate chains, however even for ink-jet printed microbeads fabricated without MNPs and with a lower liposome concentration (approx. 3x lower concentration) the streams 
were still present (Fig. 4b). Therefore the stream formation effect comes probably directly from the ink-jet technique.

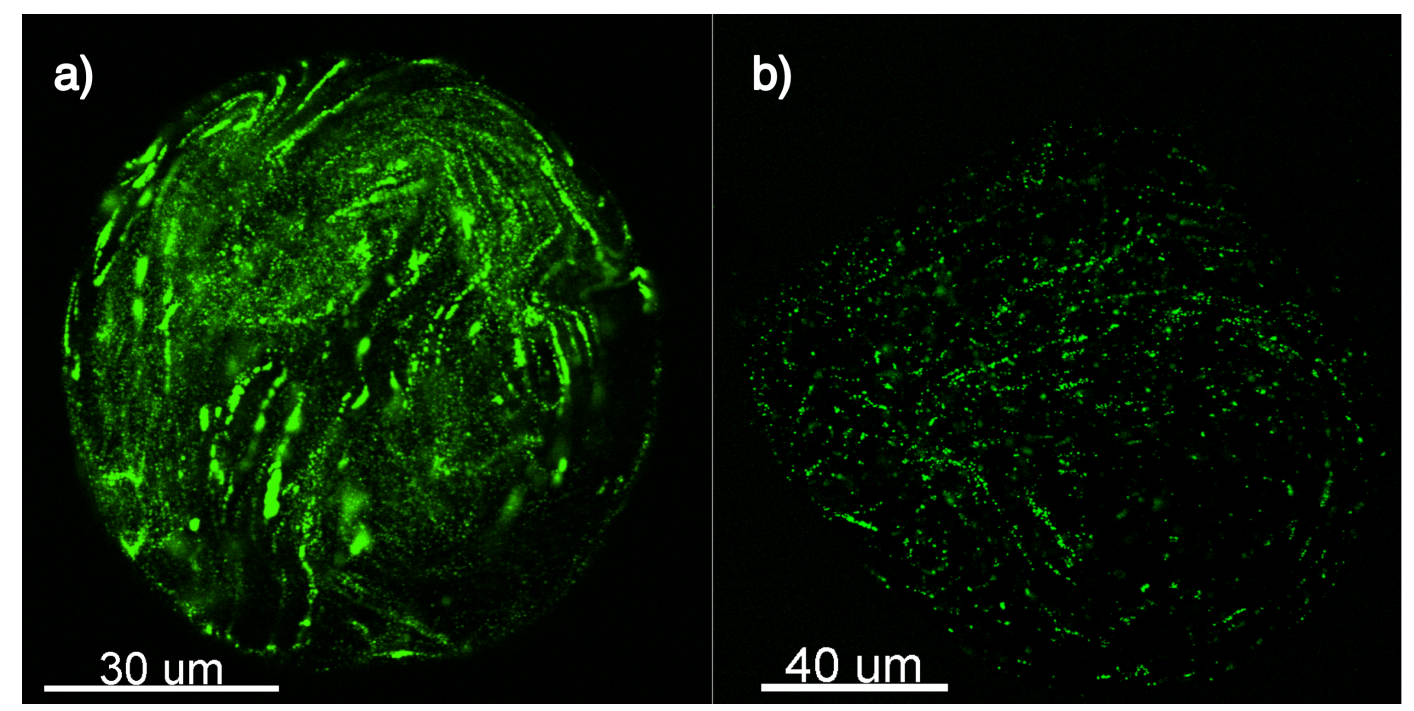

Fig. 4: Confocal fluorescent image of the LAMBs: a) standard liposome concentration, b) $3 x$ diluted liposome concentration without MNPs.

There is a strong electric field in the ink-jet print-head - in our case, the bipolar wave of a $120 \mathrm{~V}$ amplitude combined with a $80 \mu \mathrm{m}$ diameter orifice. It is known that slightly negatively charged liposomes move under the influence of external electric field ${ }^{26}$ with typical values of electrophoretic mobility of around $-5 \mu \mathrm{m} \mathrm{cm} \mathrm{V}^{-1} \mathrm{~s}^{-1}$ and that the electrophoretic mobility of liposomes is a complex characteristic influenced by the field strength, ionic strength of the solution and liposome composition. ${ }^{27}$ So the observed streams in LAMBs can be caused by the attraction/concentration of liposomes to sections of the piezoelectric element in the ink-jet nozzle during the droplet formation and subsequential internal flow within the ejected droplet coupled with the ionic gelation process.

In order to check the electrophoretic mobility of liposomes used for the LAMBs fabrication, their surface charge was measured in Tris-HCL 5mM using the ZetaSizer Nano. The calculated electrophoretic mobility was $-0.28 \pm 0.09 \mu \mathrm{mcmV}^{-1} \mathrm{~s}^{-1}$. If we consider the typical ink-jetting frequency of $200 \mathrm{~Hz}$ and the length-scale of the capillary orifice $(80 \mu \mathrm{m})$, we obtain the speed needed for the liposomes to cover this distance to be $1.6 \mathrm{~cm} / \mathrm{sec}$. From the measured electrophoretic mobility we obtain the actual average speed of liposomes in the printhead electric field as $0.4 \mathrm{~cm} / \mathrm{sec}$, therefore at least a considerable part of liposomes in the droplet can be attracted by the electric field and it is possible that the observed streams/aggregates originate in this phenomenon. Nevertheless, the electric field in the ink-jet 
capillary does not massively destabilize the liposomes as will be apparent from the release experiments discussed below.

\subsection{Release of carboxyfluorescein from $L A M B S$}

Our recent $\operatorname{study}^{22}$ on encapsulation stability of liposomes with different compositions entrapped within macroscopic alginate beads confirmed that cholesterol and low temperature can prevent the leakage of encapsulated molecules during the alginate cross-linking process. Therefore we have prepared liposomes with DPPC:Chol ratio 2:1 and used an ice-cooled bath for the $\mathrm{CaCl}_{2}$ solution in which the droplets from the print-head were cross-linked and collected. Leakage from LAMBs in an ice-cooled $\mathrm{CaCl}_{2}$ bath during the preparation and cross-linking was found to be less then $10 \%$ after $2 \mathrm{~h}$ of gelation, in accordance with results for macroscopic beads. ${ }^{22}$ The release of carboxyfluorescein from LAMBs was then studied via a temperature ramp using a heating rate of $0.1{ }^{\circ} \mathrm{C} / \mathrm{min}$. As can be seen in Fig. 5, the liposomes are stable below the "melting" temperature of the lipid bi-layer (which depends on the DPPC:Chol ratio) but once this phase transition temperature is exceeded, a rapid increase in the release rate is observed.

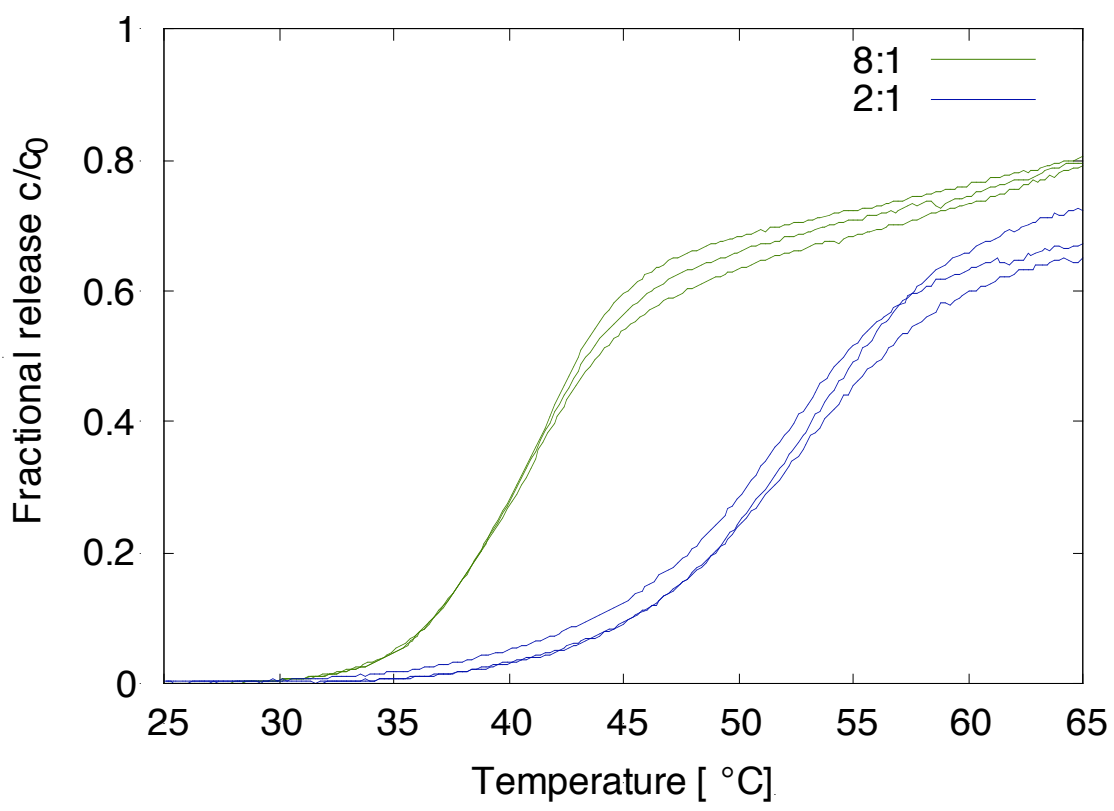

Fig. 5: Diffusion of carboxyfluorescein from LAMBs during a $0.1{ }^{\circ} \mathrm{C} / \mathrm{min}$ temperature ramp for liposomes with two DPPC:Chol ratio of 2:1 and 8:1.

The results confirm that the bilayer melting temperature for liposomes in LAMBs prepared with DPPC:cholesterol $=8: 1$ is approximately the same as for a pure DPPC bilayer (around $38{ }^{\circ} \mathrm{C}$ ). The behaviour of a cholesterol rich (DPPC:cholesterol $=2: 1$ ) mixture is more 
complex. The more cholesterol the bilayer contains, the "wider" is the transition according to DSC measurements reported $\mathrm{in}^{28}$. Moreover, the phase behavior of the lipid bilayer starts to be more complicated when more than approx. 7 mol.\% of cholesterol is present, because then there exists another lipid phase ${ }^{29}$. The phase is called cholesterol-rich and it is characterized by higher rigidity and lower permeability of the membrane. At approximately $30 \mathrm{~mol} \%$ of cholesterol the classic gel and liquid phases of the bilayer start to disappear and the melting temperature rapidly increases (see phase diagrams reported in ${ }^{29,30}$ ). It is difficult to speak about a real phase transition temperature in that kind of bilayer, however the "leakage" temperature of DPPC: cholesterol $=2: 1$ bilayer in LAMBs is between $45-50^{\circ} \mathrm{C}$, as can be seen from Fig.5.

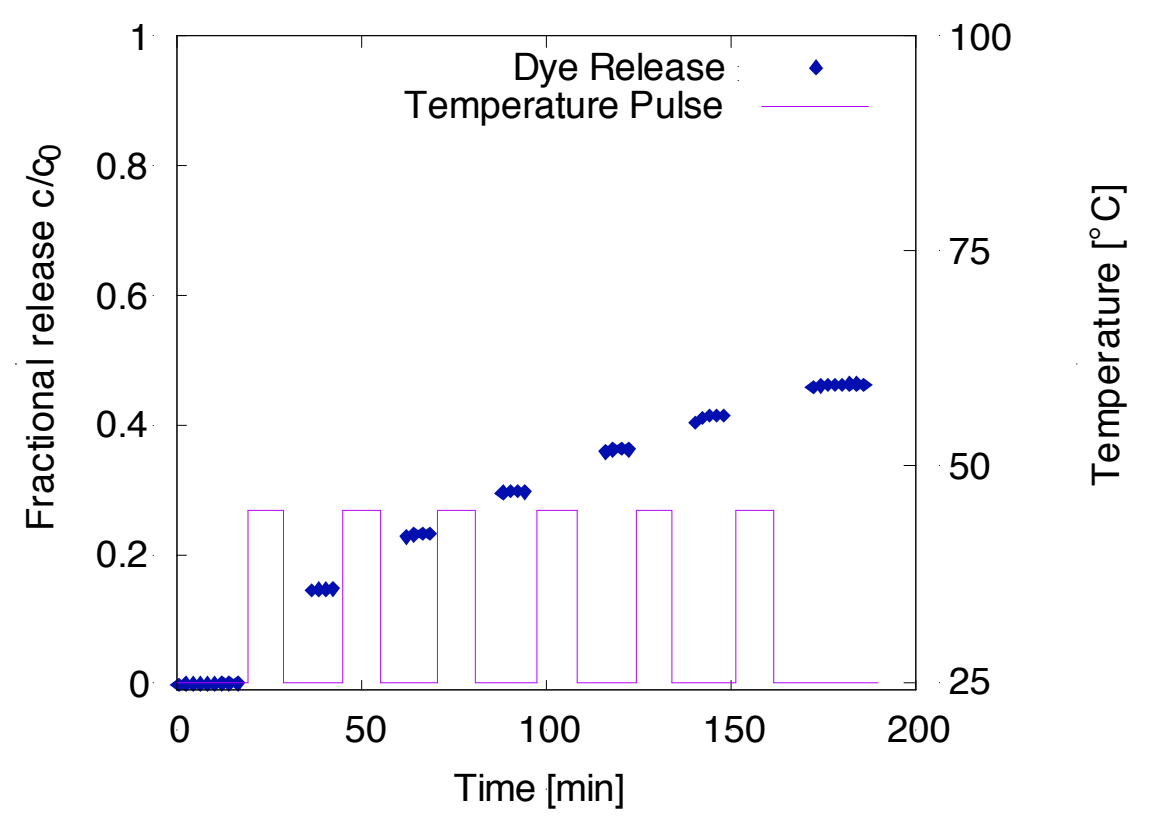

Fig. 6: Carboxyfluorescein diffusion from LAMBs during an on-off temperature program. DPPC:Chol ratio of 2:1.

In order to confirm the ability of the diffusion process to be stopped and re-started several times, piece-wise temperature-induced release experiments were carried out - see Fig. 6. After each 10-minute burst of temperature increase to $45^{\circ} \mathrm{C}$, the LAMBs were cooled back to room temperature $\left(25^{\circ} \mathrm{C}\right)$ and the change of fluorescence in the surrounding solution measured during cca 15 minutes. As Fig. 6 demonstrates, no release has occurred during the "cold" periods while each "hot" period resulted in a step increase in the quantity of released carboxyfluorescein. This means that by controlling temperature of the LAMBs, repeated ondemand release from the liposomes can be achieved, which is essential for subsequent applications such as drug delivery or local control of reaction-diffusion processes. 
The ability of magnetic nanoparticles to dissipate heat under alternating magnetic field (AMF) and therefore to induce the desired temperature change of LAMBs depends on their Specific Absorption Rate (SAR) ${ }^{31}$ defined as

$$
S A R=\frac{d T}{d t} \frac{c_{p}}{w_{M N P}}
$$

where $(d T / d t)$ is the slope of the temperature vs. time curve during the initial linear section, $c_{p}$ is the specific heat capacity of the medium (assumed equal to that of water in this work), and $w_{M N P}$ is the mass fraction of the magnetic nanoparticles in the sample. Since the SAR is known to depend on the viscosity of the surrounding medium, it was first evaluated for pure MNPs dispersed in deionized water as well as for MNPs entrapped within macroscopic alginate blocs. SAR was calculated for several MNPs concentrations $(12.5 \%, 25 \%, 50 \%$, and $100 \%$ being $7 \mathrm{~g} / 1$ ) and the results averaged to obtain SAR $390 \pm 13 \mathrm{~W} / \mathrm{g}$ for free MNPs in water and $\mathrm{SAR}=352 \pm 33 \mathrm{~W} / \mathrm{g}$ for the MNPs dispersed in the alginate blocks. The somewhat lower SAR obtained in the alginate case can be explained by the reduced mobility of the MNPs in the gel and therefore lower contribution of the Brownian relaxation mechanism. ${ }^{32}$ Nevertheless, values of SAR in the 300's W/g range are considered very good for iron oxide.

\subsection{Diffusion controlled by AMF}

As the LAMBs respond very well to static magnetic field, they were isolated from the suspension using an ordinary magnet in order to achieve a defined $100 \%$ concentration for the release experiments, which corresponds to a dense close packing of the microgel beads. These concentrated LAMBs were then diluted to $50 \%$ by deionized water and the release kinetics of $\mathrm{CF}$ as function of the applied alternating magnetic field was measured. Similarly as in the case of ordinary heating reported in Section 3.2 above, on-off AMF pulses of different duration ranging from 5 to $20 \mathrm{~min}$ were applied in order to demonstrate the ability to control the diffusion process. The results are summarized in Fig. 7 and as can be seen, the quantity released by an individual AMF pulse depends not only on the duration of the pulse but also on the "age" of the LAMB, i.e. on the remaining concentration difference between the inside and the outside of the liposomes. Although the duration of the pulse has to be increased towards the end in order to release the same quantity of $\mathrm{CF}$, the system is able to release a defined quantity of $\mathrm{CF}$ even after 10 on-off cycles (7b), which is distinctively different from typical one-off release bursts known from traditional triggered release systems. 

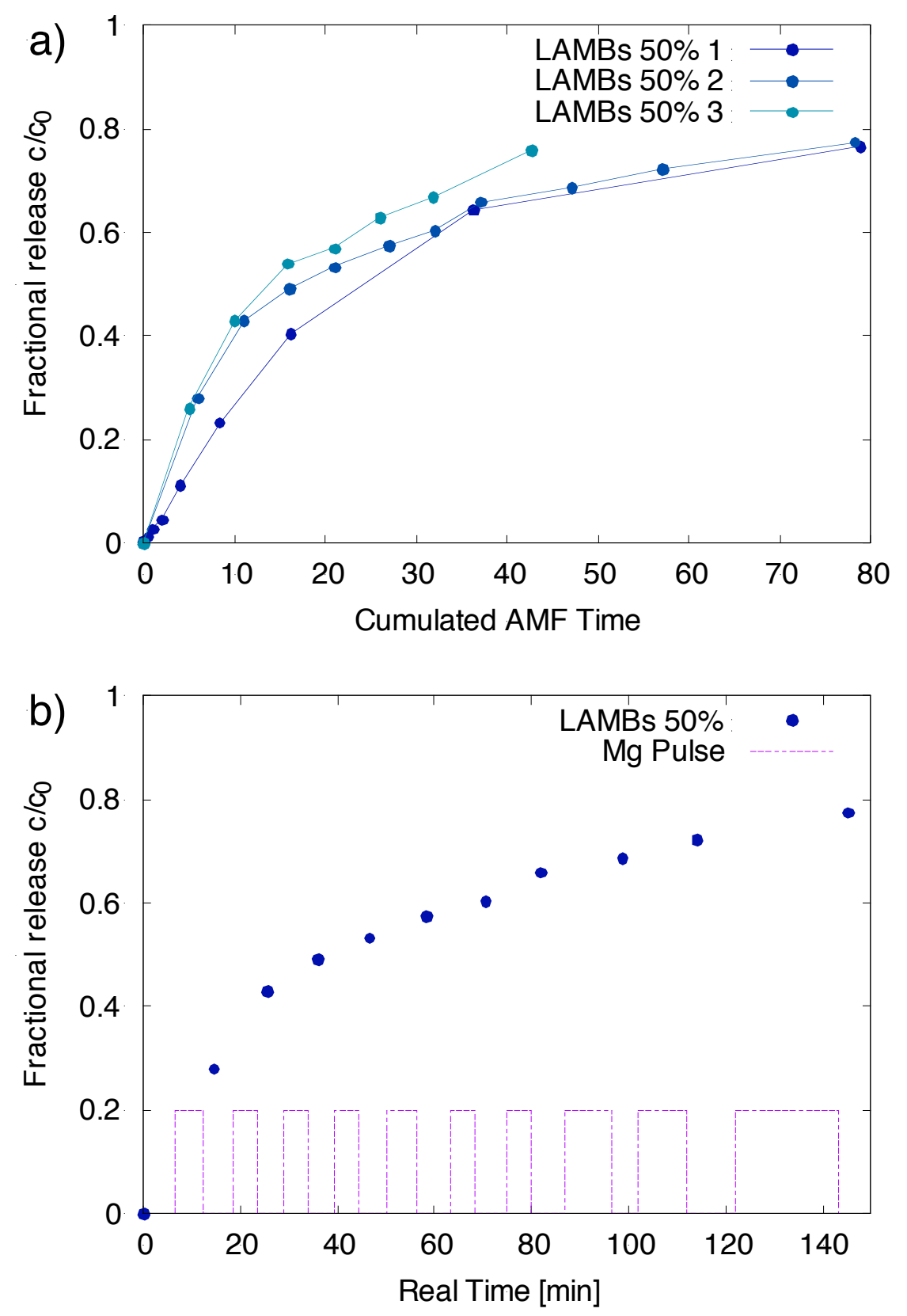

Fig. 7: Alternating magnetic field (AMF) induced diffusion of carboxyfluorescein from LAMBs in water at $50 \%$ dilution: a) release dependence on cumulated (total) AMF heating time for three experiments with different AMF pulse patterns, b) typical release for one AMF pattern in real time.

When the same experiment was repeated with diluted LAMBs (approximately 1\%), no release was observed after the application of AMF when starting from the base temperature of $25{ }^{\circ} \mathrm{C}$. Based on the previously measured temperature ramps, the release should start at approximately $40-45^{\circ} \mathrm{C}$, which may not be reachable by AMF heating for the diluted system. Therefore, the experiment was repeated from a base temperature of $37{ }^{\circ} \mathrm{C}$ but even in this 
case no release was detected. Therefore, the relationship between the LAMBs concentration and the achievable temperature rise seems to be critical for the functionality of the AMF triggered release.

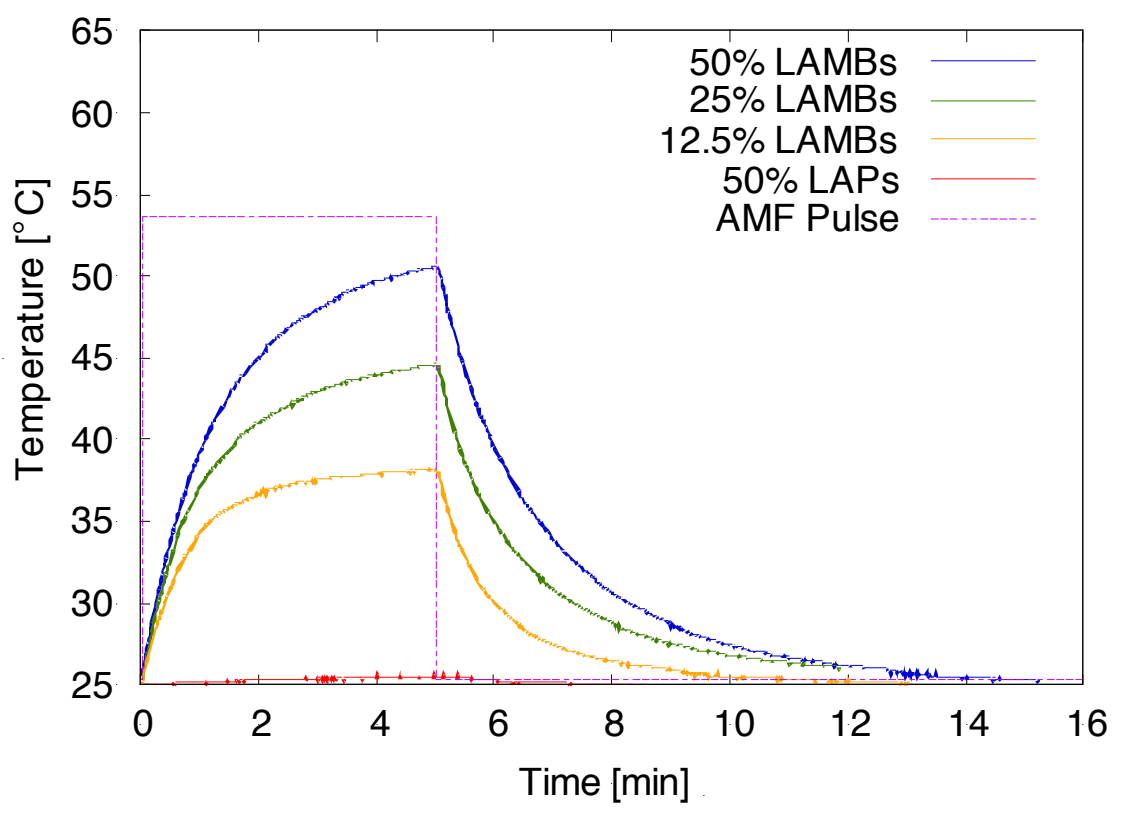

Fig. 8: Concentration dependence of temperature rise and decay upon a single 5 min pulse of alternating magnetic field (AMF). Control experiment with alginate beads prepared without magnetic nanoparticles is also shown (50\% LAPs).

Figure 8 shows the dependence of temperature on time during the application of AMF for varying LAMBs concentrations. As can be seen, in order to reliably exceed the phase transition temperature of the lipid bi-layer when starting from room temperature, a concentration of at least $25 \%$ has to be used. Fig. 8 also illustrates that as soon as the AMF is switched off, temperature quickly drops back below the phase transition regardless of the LAMBs concentration due to heat losses to the surroundings. Thus, the on-off control can be rapid in both parts of the cycles, without significant lag periods. 


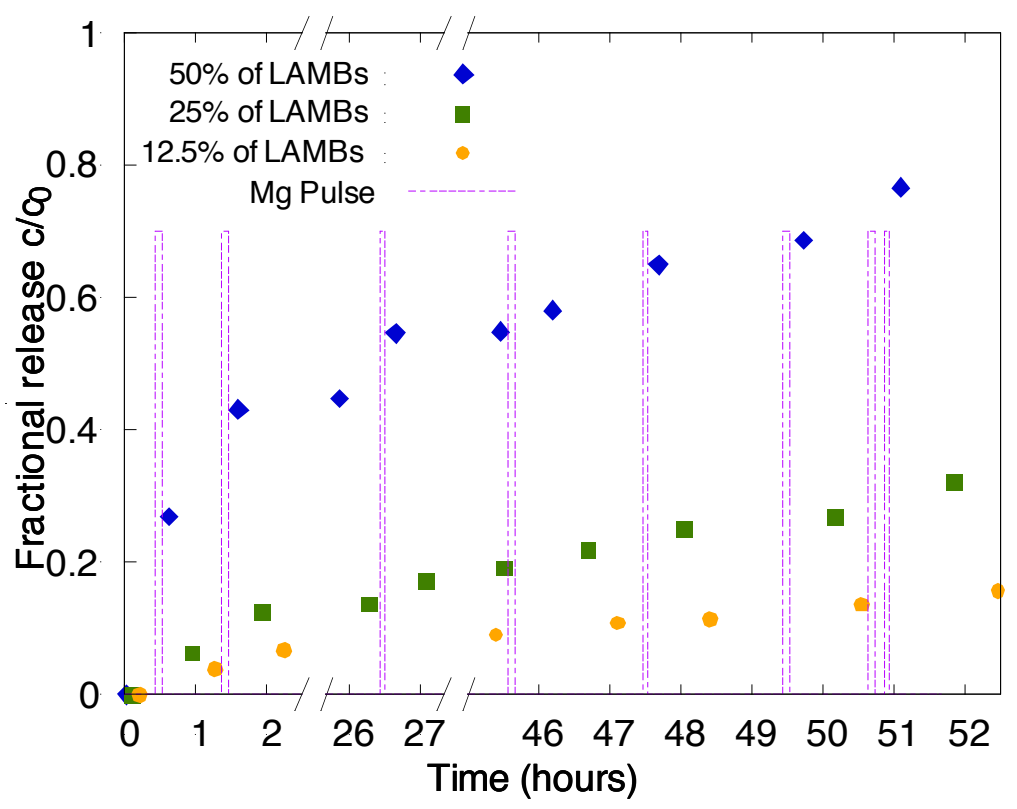

Fig. 9: AMF induced release from LAMBs at various concentrations for an arbitrary on-off sequence with long lags in between, demonstrating the stability of the LAMBs against undesired leakage when AMF is off.

The concentration dependence of AMF induced release kinetics over longer periods of time is summarized in Fig. 9. Release from concentrated LAMBs can be well controlled by the AMF pulses and at the same time no leakage is observed during the periods when AMF is off. However, the release from diluted LAMBs is only very weak (only $14 \%$ of content was released after 8 pulses of 5 minutes for the $12.5 \%$ LAMBs while concentrated $50 \%$ LAMBs released already $77 \%$ of carboxyfluorescein payload).

The temperature increase in LAMBs depends on the dynamic balance between heat generation by MNPs and heat loss to the surrounding environment. Considering the typical diameter of a single LAMB of $50 \mu \mathrm{m}$, MNP concentration in the LAMB of $3.5 \mathrm{~g} / 1$ and the SAR of $352 \mathrm{~W} / \mathrm{g}$, the heat generation rate within a single LAMB is $8.1 \times 10^{-8} \mathrm{~W}$ (approximating the LAMB as a sphere, it contains on average of $2.3 \times 10^{-10} \mathrm{~g}$ MNPs). If the LAMB is placed in stagnant water (thermal conductivity $\lambda=0.58 \mathrm{~W} \mathrm{~m}^{-1} \mathrm{~K}^{-1}$ ), the Nusselt number based on the Ranz-Marshall correlation is $N u=2$ and the heat transfer coefficient is $h$ $=2.4 \times 10^{4} \mathrm{~W} \mathrm{~m}^{-2} \mathrm{~K}^{-1}$. Using the standard heat transfer formula for $Q^{\prime}=h A \Delta T$, the asymptotic temperature difference between the LAMB and the surrounding fluid for which the heat losses are equal to the heat source is a negligible $\Delta T=4.4 \times 10^{-4}{ }^{\circ} \mathrm{C}$. For comparison, if the same LAMB had a diameter of $5 \mathrm{~mm}$ and were surrounded by a medium with a lower thermal conductivity such as air $\left(\lambda=0.024 \mathrm{~W} \mathrm{~m}^{-1} \mathrm{~K}^{-1}\right)$ rather than water, the temperature rise 
would be $107^{\circ} \mathrm{C}$. From the above calculations, it follows that local clustering of LAMBs into larger agglomerates with correspondingly lower surface/volume ratio is essential for their proper functionality.

\section{Conclusions}

The conditions enabling repeated on/off control of diffusion from liposomes embedded in a hydrogel matrix by means of radiofrequency heating of iron oxide nanoparticles have been investigated. A temperature increase above the phase transition of the phospholipid bi-layer is a necessary condition for the functionality of the system. In spite of high SAR values of the nanoparticle used, the rate of heat loss by conduction from an individual microgel bead is too high to allow any significant temperature rise to take place. The required temperature rise can only be achieved in cases where the beads are more concentrated or accumulated in a small volume. Such local concentration of beads could be achieved e.g. by the application of a static magnet, by specific adhesion to the target substrate ${ }^{33}$ or by controlled agglomeration between the beads. The advantage of the liposome-alginate-magnetite beads described in this work is that their surface can be further functionalized e.g. by sol-gel deposition of a think mesoporous silica layer as described $\mathrm{in}^{23}$, which gives scope for further control over the aggregation and adhesion processes of the microgel beads.

\section{Acknowledgments}

Support from the European Research Council (project no. 200580-Chobotix) and the European Union $7^{\text {th }}$ Framework Program (grant agreement no. 318671 - MICREAgents) is gratefully acknowledged.

\section{Supporting Information Available}

More information regarding the magnetic nanoparticles (TEM photographs, XRD patterns, size distribution and DLS data) are available as Supporting Information. This information is available free of charge via the Internet at http://pubs.acs.org/. 


\section{References}

(1) Musacchio, T.; Torchilin, V. P., Recent developments in lipid-based pharmaceutical nanocarriers. Front. Biosci. 2011, 16, 1388-412.

(2) Immordino, M. L.; Dosio, F.; Cattel, L., Stealth liposomes: review of the basic science, rationale, and clinical applications, existing and potential. Int. J. Nanomedicine 2006, 1, 297315.

(3) Chen, Y.; Bose, A.; Bothun, G. D., Controlled Release from Bilayer-Decorated Magnetoliposomes via Electromagnetic Heating. ACS Nano 2010, 4, 3215-3221.

(4) Amstad, E.; Kohlbrecher, J.; Müller, E.; Schweizer, T.; Textor, M.; Reimhult, E., Triggered Release from Liposomes through Magnetic Actuation of Iron Oxide Nanoparticle Containing Membranes. Nano Lett. 2011, 11, 1664-1670.

(5) Wu, G.; Mikhailovsky, A.; Khant, H.; Fu, C.; Chiu, W.; Zasadzinski, J., Remotely triggered liposomal release by near-infrared light absorption via hollow gold nanoshells. $J$. Am. Chem. Soc. 2008, 130, 8175-8177.

(6) Kulin, S.; Kishore, R.; Helmerson, K.; Locascio, L., Optical Manipulation and Fusion of Liposomes as Microreactors. Langmuir 2003, 19, 8206-8210.

(7) Ganta, S.; Devalapally, H.; Shahiwala, A.; Amiji, M., A review of stimuli-responsive nanocarriers for drug and gene delivery. J. Controlled Release 2008, 126, 187-204.

(8) Al-Jamal, W. T.; Kostarelos, K., Liposomes: from a clinically established drug delivery system to a nanoparticle platform for theranostic nanomedicine. Acc. Chem. Res. 2011, 44, 1094-104.

(9) Lu, A. H.; Salabas, E. L.; Schuth, F., Magnetic nanoparticles: synthesis, protection, functionalization, and application. Angew. Chem. 2007, 46, 1222-1244.

(10) Gao, J.; Gu, H.; Xu, B., Multifunctional Magnetic Nanoparticles: Design, Synthesis, and Biomedical Applications. Acc. Chem. Res. 2009, 42, 1097-1107.

(11) Sun, C.; Lee, J. S.; Zhang, M., Magnetic nanoparticles in MR imaging and drug delivery. Adv. Drug Delivery Rev. 2008, 60, 1252-65.

(12) Kumar, C. S. S. R.; Mohammad, F., Magnetic nanomaterials for hyperthermia-based therapy and controlled drug delivery. Adv. Drug Delivery Rev. 2011, 63, 789-808.

(13) Preiss, M. R.; Bothun, G. D., Stimuli-responsive liposome-nanoparticle assemblies. Expert Opin. Drug Deliv. 2011, 8, 1025-1040.

(14) Soenen, S. J.; Velde, G. V.; Ketkar,Atre, A.; Himmelreich, U.; De Cuyper, M., Magnetoliposomes as magnetic resonance imaging contrast agents. Wiley Interdiscip. Rev. Nanomed. Nanobiotechnol. 2011, 3, 197-211. 
(15) Bealle, G.; Di Corato, R.; Kolosnjaj-Tabi, J.; Dupuis, V.; Clement, O.; Gazeau, F.; Wilhelm, C.; Menager, C., Ultra magnetic liposomes for MR imaging, targeting, and hyperthermia. Langmuir 2012, 28, 11834-42.

(16) Nappini, S.; Bombelli, F. B.; Bonini, M.; Norden, B.; Baglioni, P., Magnetoliposomes for controlled drug release in the presence of low-frequency magnetic field. Soft Matter 2010, $6,154-162$

(17) Tai, L.-A.; Tsai, P.-J.; Wang, Y.-C.; Wang, Y.-J.; Lo, L.-W.; Yang, C.-S., Thermosensitive liposomes entrapping iron oxide nanoparticles for controllable drug release. Nanotechnology 2009, 20, 135101.

(18) Amstad, E.; Gillich, T.; Bilecka, I.; Textor, M.; Reimhult, E., Ultrastable Iron Oxide Nanoparticle Colloidal Suspensions Using Dispersants with Catechol-Derived Anchor Groups. Nano Lett. 2009, 9, 4042-4048.

(19) Isamu, T.; Hidekazu, S.; Toshihisa, Y., Application of Alginate Gel as a Vehicle for Liposomes. I.Factors Affecting the Loading of Drug-Containing Liposomes and Drug Release. Chem. Pharm. Bull. 1996, 44, 1941-1947.

(20) Dhoot, N. O.; Wheatley, M. A., Microencapsulated liposomes in controlled drug delivery: Strategies to modulate drug release and eliminate the burst effect. J. Pharm. Sci. 2003, 92, 679-689.

(21) Hong, Y.; Lee, H.; Kim, J.-C., Alginate beads containing pH-sensitive liposomes and glucose oxidase: glucose-sensitive release. Colloid Polym. Sci. 2009, 287, 1207-1214.

(22) Ullrich, M.; Hanus, J.; Dohnal, J.; Stepanek, F., Encapsulation stability and temperaturedependent release kinetics from hydrogel-immobilised liposomes. J. Colloid Interface Sci. 2013, 394, 380-385.

(23) Haufova, P.; Dohnal, J.; Hanus, J.; Stepanek, F., Towards the inkjet fabrication of artificial cells. Colloids Surf., A 2012, 410, 52-58.

(24) Massart, R., Preparation of aqueous magnetic liquids in alkaline and acidic media. IEEE Trans. Magn. 1981, 17, 1247-1248.

(25) Dohnal, J.; Stepanek, F., Inkjet fabrication and characterization of calcium alginate microcapsules. Powder Technol. 2010, 200, 254-259.

(26) Pysher, M. D.; Hayes, M. A., Examination of the Electrophoretic Behavior of Liposomes. Langmuir 2004, 20, 4369-4375.

(27) Chen, S. B., Electrophoretic mobility of a spherical liposome. J. Colloid Interface Sci. 2010, 348, 177-182.

(28) McMullen, T. P.; Lewis, R. N.; McElhaney, R. N., Differential scanning calorimetric study of the effect of cholesterol on the thermotropic phase behavior of a homologous series of linear saturated phosphatidylcholines. Biochemistry 1993, 32, 516-522. 
(29) Vist, M. R.; Davis, J. H., Phase equilibria of cholesterol/dipalmitoylphosphatidylcholine mixtures: deuterium nuclear magnetic resonance and differential scanning calorimetry. Biochemistry 1990, 29, 451-464.

(30) Sankaram, M. B.; Thompson, T. E., Cholesterol-induced fluid-phase immiscibility in membranes. Proc. Natl. Acad. Sci. USA 1991, 88, 8686-8690.

(31) Mornet, S.; Vasseur, S.; Grasset, F.; Veverka, P.; Goglio, G.; Demourgues, A.; Portier, J.; Pollert, E.; Duguet, E., Magnetic nanoparticle design for medical applications. Prog. Solid State Chem. 2006, 34, 237-247.

(32) Rudolf, H.; Silvio, D.; Robert, M. 1.; Matthias, Z., Magnetic particle hyperthermia: nanoparticle magnetism and materials development for cancer therapy. J. Phys.: Condens. Matter 2006, 18, S2919-S2934.

(33) Tokarova, V.; Pittermannova, A.; Cech, J.; Ulbrich, P.; Stepanek, F., Thermo-responsive adhesion properties of composite hydrogel microcapsules. Soft Matter 2012, 8, 1087-1095. 